\title{
ENFERMEDAD DE GAUCHER
}

\author{
GAUCHER DISEASE
}

Magaly Mendoza-Quispe

\begin{abstract}
RESUMEN
La enfermedad de Gaucher (EG), enfermedad autosómica recesiva, es la más frecuente del grupo de las enfermedades de depósito lisosomal. Los síntomas y signos son multisistémicos, se establecen de manera crónica y progresiva y se deben a la acumulación de glucocerebrósidos en la médula ósea, el bazo, el hígado, los pulmones, tejido esquelético y en el cerebro. Existen tres tipos de presentación de la enfermedad: Tipo I (la más común), Tipo II y Tipo III. Es importante realizar el diagnóstico precoz porque los pacientes se pueden beneficiar de la terapia de reemplazo enzimático (TRE).
\end{abstract}

Palabras clave: Enfermedad de Gaucher; Diagnóstico; Tratamiento. (fuente: DeCS BIREME)

\begin{abstract}
Gaucher's disease (GD), an autosomal recessive disease, is the most frequent of the group of lysosomal storage diseases. The symptoms and signs are multisystemic, are established chronically and progressively and are due to the accumulation of glucocerebrosides in the bone marrow, spleen, liver, lungs, skeletal tissue and in the brain. There are three types of disease presentation: Type I (the most common), Type II and Type III. It is important to make an early diagnosis because patients can benefit from enzyme replacement therapy (ERT).
\end{abstract}

Key words: Gaucher disease; Diagnosis; Treatment. (source: MeSH NLM)

\section{INTRODUCCIÓN}

La enfermedad de Gaucher (EG), debe su nombre por haber sido descrita por Phillipe Gaucher en 1882. Es la enfermedad más frecuente del grupo de las enfermedades de depósito lisosomal comprendidas dentro de los errores innatos del metabolismo'. La enfermedad de Gaucher se debe a mutaciones en el gen responsable de la síntesis de la enzima lisosomal b-glucocerebrosidasa ácida, también llamada ß-Glucosidasa ácida, (o ß-GA), cuyos locus se ubica en 1q21. El patrón de herencia es autosómico recesivo, es decir que la mutación en éste gen debe darse en estado de homocigocia ${ }^{2}$. La deficiencia enzimática favorece la acumulación de la glucosil-ceramida en los lisosomas de macrófagos (células de Gaucher) y en los monocitos del sistema monocito macrófago, llevando a la acumulación de glucocerebrósidos en la médula ósea, el bazo, el hígado, los pulmones, tejido esquelético y en el cerebro, causando daño celular y disfunción orgánica.

La prevalencia de la enfermedad de Gaucher es de 1/40 000 a 1/60 000 personas, salvo en la población de judíos Ashkenazi, en quienes es cien veces mayor. La frecuencia de portadores (heterocigotos) es de $1 / 14$ en personas con ancestros judíos Ashkenazi y de $1 / 500$ en la población general ${ }^{3}$.

\section{Signos y síntomas}

Como consecuencia, las características de la enfermedad de Gaucher, son el desarrollo de síntomas y signos multisistémicos que se establecen de manera crónica y progresiva, tales como:

Departamento de Hematología Clínica - Hospital Nacional Edgardo Rebagliati Martins, EsSalud. 
visceromegalias, destrucción ósea y citopenias periféricas ${ }^{4}$. El espectro clínico de la enfermedad es altamente heterogéneo, incluyendo adultos asintomáticos en los que se diagnostica la enfermedad de manera incidental, hasta formas muy severas como el hidrops fetalis y formas de severidad variable en la edad adulta, es posible observar esta heterogeneidad incluso en hermanos y también en gemelos ${ }^{5}$.

\section{Diagnóstico}

Hasta 1980 el diagnóstico de esta enfermedad era realizado únicamente a partir de datos morfológicos, esto es, identificar histológicamente las células de Gaucher. Estas células, que no son patognomónicas, se ven con relativa facilidad en fresco en aspirados de bazo y de médula ósea.

Por su parte, la determinación de la actividad deficiente de la enzima glucocerebrosidasa $ß$, resulta fundamental para alcanzar su diagnóstico. Esta actividad deficiente se puede dosificar en leucocitos y cultivos de fibroblastos. Las pruebas pueden hacerse en sangre completa o en gotas de sangre seca, una técnica que permite el estudio con poca cantidad de muestra y en centros de referencia que pueden estar muy lejos del paciente. Los sujetos afectados se distinguen muy bien de los portadores y de los sujetos normales por la disminución de actividad enzimática, constituyendo un método seguro para el diagnóstico de la enfermedad. En el caso de los portadores y los sujetos normales la situación es diferente, pues se produce un solapamiento entre actividad de portadores y no portadores que no permite establecer la distinción por este procedimiento. Tampoco permite distinguir entre los diferentes tipos de la enfermedad, ya que todos los pacientes tienen actividad por debajo de $10 \%$. Ahora bien, existen determinados marcadores bioquímicos con capacidad para mantener un seguimiento de la actividad de la enfermedad, como la enzima quitotriosidasa que es útil siempre que el paciente no presente una deficiencia congénita (6\% de la población general la padece). Esta enzima se produce en macrófagos activados y su actividad está incrementada en el suero de pacientes con enfermedad de Gaucher.

Otra de las pruebas complementarias útiles en el diagnóstico de enfermedad de Gaucher, es la determinación de la fosfatasa ácida resistente al tartrato, la cual se incrementa en las células de Gaucher. También, la enzima convertidora de angiotensina (ACE), que aumenta en los pacientes con enfermedad de Gaucher. Por último, la fosfatasa alcalina que puede elevarse en aquellos pacientes con afección ósea.

\section{Tipos}

Existen tres tipos de presentación de la enfermedad: Tipo I, Tipo II y Tipo III.

- Tipo I, No neuropática. Es la forma más común y afecta a 1 de cada 40000 a 60000 niños nacidos vivos, no afecta el cerebro o al sistema nervioso central, inclusive se ha visto que algunos pacientes carecen de síntomas representativos, mientras que otros desarrollan síntomas severos que pueden ser fatales.

- Tipo Il, Neuropática aguda. La enfermedad de Gaucher Tipoll es más rara y poco frecuente por eso se presenta en menos de 1 en 100000 niños nacidos vivos. Sin embargo, las personas con EG Tipo II sufren efectos más severos que las que presentan EG Tipo I, debido a que pueden sufrir grandes problemas neurológicos en conjunto con la anemia, trombocitopenia y fuertes dolores por las crisis óseas características de la enfermedad, ocasionando que muchos de ellos no vivan pasados los dos años de edad.

- Tipo III. Neuropática crónica. El Tipo III es también poco común y afecta a menos de 1 en 100000 niños nacidos vivos. Esta forma de la enfermedad también puede causar signos y síntomas neurológicos, pero estos son menos severos que los de la EG Tipo II. Los signos y síntomas aparecen durante la infancia o niñez, y los pacientes con la enfermedad de Gaucher Tipo III pueden vivir pasada la edad adulta.

\section{Evolución y complicaciones}

Es importante realizar el diagnóstico precoz de la enfermedad de Gaucher, porque los pacientes se pueden beneficiar de la terapia de reemplazo enzimático (TRE), y prevenir de esta manera el desarrollo de complicaciones irreversibles, como la necrosis avascular articulary el retraso en el crecimiento que tiene implicancias en la calidad de vida6. Las manifestaciones clínicas de las esfingolipidosis no se explican únicamente por una deficiencia cuantitativa de enzimas ya que con solo $10-20 \%$ de la actividad residual de la enzima es suficiente para llevar una vida normal, por lo que se requiere la interacción de otros mecanismos para que se den estas manifestaciones clínicas ${ }^{7}$. La presentación puede ser en infantes (los de peor pronóstico), jóvenes o adultos; no siempre hay asociación entre la expresión clínica de la enfermedad y las mutaciones genéticas que se han identificado ${ }^{8}$. Entre 10 a $25 \%$ de los pacientes son asintomáticos y pueden ser diagnosticados a partir del estudio familiar de un miembro afectado9,10. La afectación ósea es la manifestación clínica más frecuente y la que produce mayores secuelas; como parte de la enfermedad ósea, 
se presenta infiltración de la medula ósea por las células de Gaucher, que pueden estimular la fibrosis, necrosis, infartos, lesiones líticas, osteopenia, deformidades y fracturas patológicas, hechos que se evidencian mediante biopsia de medula ósea y radiológicamente, constituyéndose ambos en un método diagnostico ${ }^{11}$.

La afectación visceral principalmente del bazo y su crecimiento se correlacionan con la progresión de la enfermedad, sus manifestaciones de citopenia, principalmente trombocitopenia son documentadas por imágenes y exámenes de sangre ${ }^{12}$. La hepatomegalia es menor con excepción de los pacientes esplenectomizados, donde puede haber fibrosis y cirrosis, hasta hematopoyesis extramedular ${ }^{4}$.

\section{Manejo y tratamiento de la enfermedad}

\section{Tipos de tratamiento:}

1.Terapia de reemplazo enzimático (TRE): Existen tres drogas aprobadas por la FDA: Imiglucerasa, velaglucerasa y taliglucerasa. En nuestro país sólo está disponible comercialmente la imiglucerasa, para su uso en enfermedad de Gaucher.

2.Terapia de reducción del sustrato: Miglustat, eliglustat, fármacos aún no disponibles comercialmente en nuestro país.

Si bien, entre las posibles opciones terapéuticas, la que mejores perspectivas ha mostrado, por el momento, es la terapia de reemplazo enzimático (TRE) con Imiglucerasa. Desde su aprobación en 1991 por la FDA, su uso en la práctica clínica habitual ha modificado la historia natural de esta enfermedad. En su inicio se obtenía a partir de placentas humanas y posteriormente a partir de tecnología del ácido desoxirribonucleico (ADN) recombinante, siendo el nombre de su principio activo imiglucerasa ${ }^{12}$.

Actualmente, se ha demostrado una alta eficacia en el control y evolución de la ${ }^{7}$. De este modo, la disminución de las visceromegalias se observa en 1 a 3 años, las alteraciones celulares plasmáticas se resuelven en 1 a 5 años y las lesiones óseas reversibles como osteopenia y osteoporosis mejoran en una media de dos años. Algunas lesiones focales en los adultos también mejoran. La terapia de reemplazo enzimático ha sido especialmente efectiva entre pacientes pediátricos con afectación ósea, ya que no sólo puede detener la progresión de la enfermedad sino también inducir a la regresión de algunas de las lesiones preexistentes ${ }^{9}$. Actualmente, se ha establecido una dosificación individual según la extensión de la enfermedad. En este sentido, una vez conocido el grado de afectación orgánica, se deben establecer los objetivos terapéuticos para en base a ello establecer la dosis óptima. En niños, se recomienda siempre iniciar el tratamiento con dosis no superiores a 40 a $60 \mathrm{U} / \mathrm{kg}$. Su continuo estado de crecimiento implica modular la dosis, así como también para evitar el agravamiento de las lesiones óseas, ya que con la aplicación del fármaco a dosis altas se corre el riesgo de empeorar las lesiones óseas ya existentes ${ }^{10}$. Habitualmente, se administra una dosis cada quince días, con evaluaciones periódicas (según el consenso elegido para su seguimiento) para realizar los ajustes de dosis necesarios. El tratamiento desde su inicio tiene carácter indefinido dado que, según la evidencia científica, suspensiones repentinas en el tratamiento pueden resultar perjudiciales con la consiguiente reaparición de los signos de la enfermedad.

Tabla 1. Terapia de reemplazo enzimático (TRE).

\section{OBJETIVOS GENERALES}

- Normalizar los niveles de hemoglobina.

- Mejorar el recuento de plaquetas alcanzando niveles seguros.

- Reducir la visceromegalia hasta un nivel en el que ya no provoque síntomas.

- Prevenir la necesidad de una esplenectomía.

- Prevenir las crisis óseas y las fracturas.

- $\quad$ Promover un crecimiento óptimo. 


\section{CONCLUSIÓN}

la enfermedad de Gaucher es la enfermedad de depósito lisosomal más común; por deficiencia de la enzima ß-Glucosidasa ácida se acumulan glucocerebrósidos en la médula ósea, bazo, hígado, pulmones, tejido esquelético y a veces en el cerebro. La presentación de la enfermedad Tipo I, no neuropática, es la más común. El cuadro clínico es altamente heterogéneo y el diagnóstico precoz e inicio de la terapia de reemplazo enzimático (TRE) permiten prevenir el desarrollo de complicaciones irreversibles.
Contribuciones de autoría: El autor participó en la generación, redacción y aprobación final del artículo original.

Financiamiento: Autofinanciado.

Conflicto de interés: La autora declara no tener conflicto de interés en la publicación de este artículo.

Recibido: 18 de enero del 2018

Aprobado: 22 de febrero del 2018

Correspondencia: Magaly Mendoza Quispe

Dirección: Departamento de Hematología Clínica-Hospital Nacional Edgardo Rebagliati Martins. Av. Edgardo Rebagliati 490, Jesús María, Lima - Perú 15072 Celular: $+511-987248484$

Correo:maggi_mendoza@yahoo.es

\section{REFERENCIAS BIBLIOGRÁFICAS}

1. Bohra V, Nair V. Gaucher's disease. Indian J Endocrinol Metab. 2011 15(3):182-6.

2. Yildiz $Y$, Hoffmann $P$, VomDahl $S$, et al. Functional and genetic characterization of the non-lysosomal glucosylceramidase 2 as a modifier for Gaucher disease. Orphanet J Rare Dis. 2013; 8:151.

3. Valle D, Beaudet A, Vogelstein, et al. (Editores). The Online Metabolic and Molecular Bases of Inherited Disease. The McGraw-Hill Companies 2001 (Citado 6 de octubre de 2014). Disponible en: http://ommbid. mhmedical.com/book. aspx?bookID=474

4.JmoudiakM, Futerman AH. Gaucher disease:pathological mechanisms and modern management. Br J Haematol. 2005; 129(2):178-88.

5. Van Breemen MJ, Bleijlevens B, de Koster CG, Aerts JMFG. Limitations in quantitation of the biomarker CCL18 in Gaucher disease blood samples by surface-enhanced laser desorption/ionization time-of flight mass spectrometry. Biochim Biophys Acta. 2006; 1764(10):1626-32.

6. Eblan MJ, Goker-Alpan O, Sidransky E. Perinatal lethal Gaucher disease: a distinct phenotype along the neuronopathic continuum. Fetal Pediatr Pathol. 2005; 24(4-5):205-22.
7. Giraldo P. Guía de actuación en pacientes con enfermedad de Gaucher tipo 1. Med Clínica. 2011; 137:55-60.

8. Germain DP. Gaucher's disease: a paradigm for interventional genetics. Clin Genet. 2004; 65(2):77- 86.

9. Franco-Ornelas S; Grupo de expertos de enfermedad de Gauche. Consenso Mexicano de Enfermedad de Gaucher. Rev Med Inst Mex Seguro Soc. (Internet) 2010; (Citado 6 de octubre de 2014). 48(2):167186. Disponible en: http://revistamedica.imss.gob. $\mathrm{mx} /$ index. php?option=com_multicategories\&view $=$ article\&id $=809$ :consensomexicano-de-enfermedadde-gaucher\&ltemid $=602$

10. Drelichman G, Basack N, Fernandez N, et al. Consenso para la Enfermedad de Gaucher: Grupo Argentino de diagnóstico y tratamiento de la Enfermedad de Gaucher. Hematol 2013; 17:25-60.

11. Bembi B, Ciana G, Mengel E, Terk MR, Martini C, Wenstrup RJ. Bone complications in children with Gaucher disease. Br J Radiol. 2002; 75(S1): A37-44

12. Grabowski GA,Andria G, BaldellouA, et al. Pediatric nonneuronopathic Gaucher disease: presentation, diagnosis and assessment. Consensus statements. Eur J Pediatr. 2004; 163(2):58-66.

Consulte la Versión Electrónica de la Revista:

Facultad de Medicina Humana Universidad Ricardo Palma

http://revistas.urp.edu.pe/index.php/RFMH
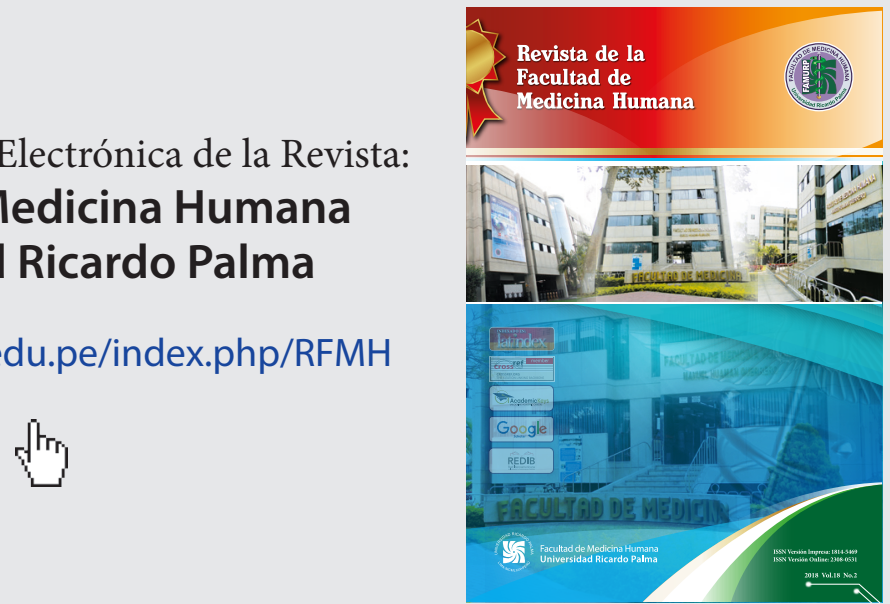